\title{
Clinical Observation of Gene Polymorphism of Olanzapine or Aprepitant in Prevention of CINV
}

\author{
Yilan Jin ${ }^{1, *}$ \\ Gaowa Jin ${ }^{2, *}$ \\ Jun Zhao ${ }^{2}$ \\ Caihong Jiang ${ }^{2}$ \\ Lanzhen Zhao ${ }^{2}$ \\ Ying Jiang ${ }^{2}$ \\ Feng Chen ${ }^{2}$ \\ Hui $\mathrm{Li}^{2}$ \\ Wenjuan Wang ${ }^{2}$ \\ Yungaowa $\mathrm{Wu}^{2}$ \\ Guang $\mathrm{Liu}^{2}$ \\ Xiaorong $\mathrm{Li}^{2}$ \\ Min $\mathrm{Gu}^{2}$ \\ Xiaomei $\mathrm{Li}^{3}$ \\ Quanfu $\mathrm{Li}^{2}$ \\ 'Ordos Clinical College, Inner Mongolia \\ Medical University, Ordos, 017000 , \\ People's Republic of China; ${ }^{2}$ Department \\ of Medical Oncology, Ordos Central \\ Hospital, Ordos, 017000, People's \\ Republic of China; ${ }^{3}$ Department of \\ Medical Oncology, The General Hospital \\ of the People's Liberation Army, Beijing, \\ 100853, People's Republic of China \\ *These authors contributed equally to \\ this work
}

Correspondence: Quanfu Li

Department of Medical Oncology, Ordos Central Hospital, Ordos, 0I7000, People's

Republic of China

Tel +86 477-8363279

Fax +86 477-8363158

Email li_qfedu@।63.com

Xiaomei Li

Department of Medical Oncology, The

General Hospital of the People's

Liberation Army, Beijing, 100853, People's

Republic of China

Tel +86 I350l308592

Email lixoamei@163.com
Objective: The present study aims to investigate the correlation between the gene polymorphisms of the multidrug resistance protein 1 (ABCB1), the intron region of transcriptional factor (GTF2E1) and catechol-O-methyltransferase (COMT), dopamine receptor (DRD2), and the control of chemotherapy-induced nausea and vomiting (CINV) by olanzapine or aprepitant in a Chinese population under a fractionated cisplatin dosing pattern.

Methods: Antiemetic treatment with $5 \mathrm{mg}$ of olanzapine or aprepitant triplet therapy was conducted in 210 patients with malignancies receiving cisplatin multi-day chemotherapy. The general data on the patients were collected with the evaluation of the rate of complete protection (TP), complete remission (CR), complete control (TC), and time to first vomiting, the functional living index-emesis (FLIE) scale, and side effects in the acute and delayed phases. The DNA mass spectrometry detected the gene polymorphisms of ABCB1, GTF2E1, COMT, and DRD2, and the correlation with TP was analyzed.

Results: 1) There were no statistically significant differences in the TP, CR, TC, time of first vomiting, and FLIE index at different phases between the $5 \mathrm{mg}$ of olanzapine group and the aprepitant group $(\mathrm{P}>0.05)$. 2) The main side effect in the olanzapine group was drowsiness $(\mathrm{P}=$ $0.00)$, and in the aprepitant group was constipation $(\mathrm{P}=0.02) .3)$ The distributions of each genotype were in the Hardy-Weinberg $(\mathrm{H}-\mathrm{W})$ equilibrium. Univariate analysis showed that in the olanzapine group, delayed-phase TP was correlated with the ABCB1 rs1045642 non-TT ( $\mathrm{P}=0.01)$ genotype. Conclusion: The present study revealed that females and the rs1045642TT genotype were independent risk factors for delayed-phase CINV in the northern Chinese population, which provided a scientific basis for subsequent CINV-related analysis of high-risk factors in Chinese patients.

Keywords: chemotherapy-induced nausea and vomiting, cisplatin, multi-day chemotherapy, gene polymorphism

\section{Introduction}

With the update of clinical guidelines and the clinical application of 5-HT 3 RA, NK1 receptor antagonist (NK-1RA) and olanzapine, the control rate of CINV has increased significantly, but at least $20-30 \%$ of the patients have experienced CINV. ${ }^{1}$ The occurrence and severity of CINV are influenced by many factors, broadly classified into therapeutic and patient-related risk factors. Among them, therapeutic factors include the type of drug used, the dose, the order and route of administration, etc. ${ }^{2,3}$ Patient-related risk factors include young age, female, previous administration of chemotherapy agents, little or no alcohol consumption, morning sickness, anxiety, etc. However, the risk factors correlated with CINV have not been fully elucidated. 
The study of the correlation between gene polymorphism and CINV is one of the hot spots in recent years, and interindividual gene profiles may be an independent risk factor for CINV. Studies concerning this area have mainly focused on European, American, and Japanese populations, and there are significant differences in gene frequencies among different ethnic groups. It is of clinical significance to explore the relationship between gene polymorphisms and CINV in the Chinese population. ${ }^{4-11}$ The gene of ATP-binding cassette transporter protein B1 $(\mathrm{ABCB} 1)$, intron region of transcriptional factor (GTF2E1), the dopamine receptor (DRD2) reported in Japanese population, and catechol- $O$-methyltransferase (COMT) may be associated with CINV. ABCB1 gene encodes drug transporter P-glycoprotein, which affects the transport of many antiemetic drugs and their metabolites. ${ }^{7}$ GTF2E1 is a necessary factor for RNA polymerase II to initiate transcription, which is essential in cellular signal pathway transcription. ${ }^{11}$ DRD2 gene polymorphism may affect the occurrence and severity of CINV by changing the folding structure of mRNA to reduce the stability of DRD2 receptor or its synthetic ability. ${ }^{11}$ And COMT is an enzyme that catalyzes the methylation of catechol substrates and usually plays a role in the metabolism of catecholamines and other drugs. ${ }^{11}$ The present study aims to investigate the correlation between these gene polymorphisms and cisplatin-induced nausea and vomiting and antiemetic efficacy in a randomized controlled trial of $5 \mathrm{mg}$ of olanzapine or aprepitant for the prevention of CINV in a fractionated cisplatin dosing pattern in a northern Chinese population.

\section{Materials and Methods}

\section{Study Subjects}

In the present study, 210 patients with malignancies who visited the Department of Medical Oncology of Erdos Central Hospital from March 2019 to December 2020 to receive cisplatin multi-day chemotherapy were selected. The patients were randomized into two groups: 104 received olanzapine combined with 5-hydroxytryptamine (5-HT3) receptor antagonist (5-HT3 RA) and dexamethasone triple antiemetic regimen, and 106 patients received aprepitant triple antiemetic regimen. The general characteristics between the two groups of patients were comparable, and the details are shown in Table 1. Informed consent was obtained from study participants. The present study was approved by the Ethics Committee of Erdos Central
Hospital and registered in the China Clinical Trials Registry (registration number: ChiCTR2000036826).

\section{The Inclusion and Exclusion Criteria}

The inclusion criteria were as follows: 1) patients aged $\geq 18$ years, with pathologically confirmed malignancy, to receive a regimen containing cisplatin, the total dose calculated as $75 \mathrm{mg} / \mathrm{m}^{2}$ divided over 3 days. 2) Patients with a Karnofsky (KPS) score of $\geq 70$ points. 3) Patients with no abnormality in liver and kidney function, routine blood work, and electrocardiogram (ECG) before chemotherapy. The results of the blood test met the following criteria: leukocytes $>3.5 \times 10^{9} / \mathrm{L}$, neutrophils $>1.5 \times 10^{9} / \mathrm{L}$, platelets $>85 \times 10^{9} / \mathrm{L}$, alkaline phosphatase and glutaminase $<2.5$ times the upper limit of the normal reference range, bilirubin and creatinine $<1.5$ times the upper limit of the normal reference range. 4) Patients with no contraindication for chemotherapy after evaluating the tumor's site by computed tomography (CT) or magnetic resonance imaging (MRI). 5) Patients with no nausea or vomiting for 1 week before the enrollment and no aprepitant or olanzapine use. 6) Patients who signed the informed consent form.

The exclusion criteria were as follows: 1) patients who could not take oral medication. 2) Patients with long-term administration of hormones. 3) Patients with concurrent radiotherapy. 4) Patients who had vomiting $24 \mathrm{~h}$ before chemotherapy. 5) Patients with uncontrolled intracranial metastases and patients who had undergone brain radiotherapy. 6) Lactating and pregnant females. 7) Concurrent use of quinolone antibiotics. 8) Patients with incomplete or complete bowel obstruction. 9) Patients with hypersensitivity to 5-HT3 receptors, aprepitant, or olanzapine. 10) Patients using prohibited drugs or alcoholic. 11) Patients with digestive tract tumors.

\section{Methods}

1) Patients enrolled in the present study were given a total cisplatin dose of $75 \mathrm{mg} / \mathrm{m}^{2}$ of body surface area, divided over 3 days. And all patients received multi-day cisplatin regimens were combined with one of the following chemotherapeutic drugs: gemcitabine, docetaxel, etoposide, pemetrexed, paclitaxel, vinorelbine and irinotecan, sometimes plus bevacizumab or rituximab, or both. The olanzapine triple antiemetic regimen was $5 \mathrm{mg}$ of olanzapine days $1-4,5 \mathrm{mg}$ of tropisetron days $1-3,10 \mathrm{mg}$ of dexamethasone days 1-3 from the day of cisplatin administration. The aprepitant triple antiemetic regimen was 
Table I Baseline Characteristics of Patients in Two Groups (n (\%))

\begin{tabular}{|c|c|c|c|}
\hline Characteristics & Olanzapine Group $(n=104)$ & Aprepitant Group $(n=106)$ & $\mathbf{P}$ \\
\hline Age (years) & $59.26 \pm 8.865$ & $60.01 \pm 10.358$ & 0.574 \\
\hline$\geq 55$ & $79(75.96)$ & 78 (73.58) & 0.692 \\
\hline Gender & & & 0.755 \\
\hline Female & $40(38.46)$ & $43(40.57)$ & \\
\hline Male & $64(61.54)$ & $63(59.43)$ & \\
\hline History of motion sickness & $17(16.35)$ & $21(19.8 I)$ & 0.514 \\
\hline History of female pregnancy vomiting & $13(32.5)$ & II (25.58) & 0.487 \\
\hline Alcohol use & & & 0.725 \\
\hline No consumption & $48(46.15)$ & $50(47.17)$ & \\
\hline$<4$ Drinks per week & $38(36.54)$ & 34 (32.08) & \\
\hline$\geq 4$ Drinks per week & $18(|7.3|)$ & $22(20.75)$ & \\
\hline Smoking index & & & 0.474 \\
\hline No smoking & $39(37.50)$ & $47(44.34)$ & \\
\hline $0-400$ & $13(12.50)$ & $9(8.49)$ & \\
\hline$\geq 400$ & $52(50.00)$ & $50(47.17)$ & \\
\hline Type of malignance & & & 0.755 \\
\hline Lung cancer & $40(38.46)$ & $43(40.57)$ & \\
\hline Others & $64(6 I .54)$ & $63(59.43)$ & \\
\hline Chemotherapy cycle & & & 0.232 \\
\hline First cycle & $27(25.96)$ & $37(34.90)$ & \\
\hline Second cycle & $30(28.845)$ & $23(21.70)$ & \\
\hline Third cycle & $17(16.35)$ & $23(21.70)$ & \\
\hline$\geq$ Fouth cycle & $30(28.845)$ & $23(21.70)$ & \\
\hline
\end{tabular}

$125 \mathrm{mg}$ of aprepitant on day $1,80 \mathrm{mg}$ days $2-3,5 \mathrm{mg}$ of tropisetron days $1-3$, and $5 \mathrm{mg}$ of dexamethasone days $1-$ 3. The previous study has shown that aprepitant interferes with dexamethasone's pharmacokinetics, so the dose of dexamethasone in the aprepitant group was reduced by half. $^{12}$ 2) On the day of chemotherapy, $5 \mathrm{~mL}$ of peripheral blood was drawn from 90 patients and stored at $-80^{\circ} \mathrm{C}$ for further analysis. Beijing Liuhe Huada Gene Technology Co. was commissioned to perform sequencing and typing of the single nucleotide polymorphism (SNP) loci of rs1045642, rs2032582, rs1128503, rs447978, rs4680, rs6277, and rs 1076560 by DNA mass spectrometry.

\section{Evaluation Indicators}

With the initiation of chemotherapy, patients were issued diaries or had daily check-ups to record the time, number, intensity, and adverse reactions of nausea and vomiting within 0-120 hours after chemotherapy. Patients were instructed to fill in the functional living index-emesis (FLIE) scale. The primary evaluation indicators were the acute-phase $(0-24 \mathrm{~h})$ and delayed-phase $(25-120 \mathrm{~h})$ total protection rate (TP). The secondary indicators were complete remission rate (CR) and complete control rate (TC) at different phases and the time of first vomiting, The FLIE scale, and the data concerning the common side effects.

TP was defined as no vomiting or severe dry vomiting requiring relief measures, with a maximum nausea score of $\leq 25 \mathrm{~mm}$ on the $100 \mathrm{~mm}$ nausea assessment scale. CR was defined as no vomiting or severe dry vomiting requiring palliative measures after chemotherapy. TC was defined as no vomiting or severe dry vomiting requiring palliative measures and a maximum nausea score of $\leq 5 \mathrm{~mm}$ on the $100 \mathrm{~mm}$ nausea assessment scale.

\section{Statistical Methods}

The SPSS 25.0 software was adopted for statistical analysis. The mean \pm standard deviation $(\bar{x} \pm \mathrm{SD})$, independent sample $t$-test, and chi-square test were used to compare the two groups' baseline characteristics. The $\chi 2$ test was used to analyze whether the genotype distribution conformed to 
the Hardy-Weinberg $(\mathrm{H}-\mathrm{W})$ law of genetic equilibrium. Univariate analysis was performed using the $\chi 2$ test, and the Fisher's exact test was used when the theoretical frequency was less than five. Binary logistic regression analysis was used for multiple factors. The Kaplan-Meier curve was used to compare the time of first vomiting. $P<0.05$ was considered statistically significant.

\section{Results}

\section{The Therapeutic Effects of CINV} Comparison of Different Evaluation Indicators

The delayed-phase TP was superior in the olanzapine group than in the aprepitant group. The acute-phase and delayed-phase TP in the olanzapine group and the aprepitant group were $94.23 \%(98 / 104)$ vs $95.45 \%(98 / 106)(P=$ $0.61)$, and $54.81 \%(57 / 104)$ vs $54.72 \%(58 / 106)(P=$ 0.99), respectively. The acute-phase and delayed-phase $\mathrm{CR}$ in the olanzapine group and the aprepitant group were $96.15 \%$ vs $97.17 \%(P=0.98), 75.00 \%$ vs $79.25 \%$ $(P=0.46)$, respectively. The acute-phase and delayedphase TC in the olanzapine group and the aprepitant group were $80.77 \%$ vs $82.08 \%$ ( $P=0.81), 31.73 \%$ vs $27.36 \%(P=0.49)$, respectively. There was no statistically significant difference in the above indicators between the two groups.

\section{Comparison of the FLIE, Time of First Vomiting, and} Side Effects

A score greater than 108 on the FLIE scale indicated no effect of CINV on the quality of life of the patients, and the percentage of patients with a score greater than 108 in the olanzapine group and aprepitant group in the present study was $63.46 \%$ vs $66.04 \%$, respectively $(P=0.96)$.

In comparing the time of first vomiting, the results indicated that the time of first vomiting was relatively later in the aprepitant group than in the olanzapine group, but the difference was not statistically significant $(P=0.56)$.

The main side effect in the olanzapine group was drowsiness $(P=0.00)$ and in the aprepitant group was constipation $(P=0.02)$. There were no statistically significant differences in the incidences of eczema, weakness, dizziness, headache, loss of appetite, or abdominal distension between the two groups $(P>0.05)$.

\section{Correlation Between the Gene Polymorphism and TP of CINV}

The distributions of each genotype were in the $\mathrm{H}-\mathrm{W}$ balance. There were 90 blood specimens; as there were duplicate patients enrolled in different chemotherapy cycles, there were a total of 191 cases. The follow-up records of 191 cases were included in the univariate and multivariate analyses. As shown in Table 2, the univariate analysis showed that in the olanzapine group, delayedphase TP was higher in those with the ABCB1 rs1045642 non-TT genotype than with the TT genotype $(P=0.01)$. In the aprepitant group, the delayed-phase TP was higher in those with the ABCB1 rs2032582 non-CC genotype than with the $\mathrm{CC}$ genotype $(P=0.01)$.

The gender, grouping, the ABCB1 rs1045642, and rs2032582 were introduced into a multivariate logistic regression model, and the results showed that females $(P=0.00)$ and the ABCB1 rs1045642TT genotype $(P=$ 0.002 ) were independent risk factors for CINV in the total or delayed-phases (Table 3).

\section{Discussion}

The present study was the first clinical study on the prevention of cisplatin multi-day chemotherapy regimen induced CINV by a triple antiemetic regimen of $5 \mathrm{mg}$ olanzapine or aprepitant. The study's CR rates were

Table 2 Univariate Analysis of TP and SNPs in Olanzapine and Aprepitant Group

\begin{tabular}{|l|l|l|l|l|l|}
\hline \multicolumn{2}{|l|}{ Polymorphism } & \multicolumn{2}{l|}{ Olanzapine Regimen (n (\%)) } & \multicolumn{2}{l|}{ Aprepitant Regimen (n (\%)) } \\
\cline { 3 - 6 } & Acute Phase & Delayed Phase & \multicolumn{2}{l|}{ Acute Phase } & Delayed Phase \\
\hline rs1045642 & TT & $17(94.4)$ & $5(27.8)$ & $6(100.0)$ & $3(50.0)$ \\
& Non-TT & $71(93.4)$ & $48(63.2)$ & $84(92.3)$ & $52(57.1)$ \\
& P & 1.00 & 0.01 & 1.00 & 1.00 \\
\hline rs2032582 & GG & $27(93.1)$ & $17(58.6)$ & $23(88.5)$ & $9(34.6)$ \\
& Non-GG & $61(93.8)$ & $36(55.4)$ & $67(94.4)$ & $46(64.8)$ \\
& P & 0.27 & 0.77 & 0.38 & 0.01 \\
\hline
\end{tabular}

Note: Only showed the significant data. 
Table 3 Multivariate Logistic Regression Analysis of TP and Clinical Variables in Acute and Delayed Phase

\begin{tabular}{|c|c|c|c|c|c|c|}
\hline \multirow[t]{2}{*}{ Clinical Factors } & \multicolumn{3}{|c|}{ Acute Phase } & \multicolumn{3}{|c|}{ Delayed Phase } \\
\hline & OR & $95 \% \mathrm{Cl}$ & $P$ value & OR & $95 \% \mathrm{Cl}$ & $P$ value \\
\hline \multicolumn{7}{|l|}{ Gender } \\
\hline Male vs Female & 0.46 & $0.14-1.48$ & 0.19 & 0.31 & $0.17-0.59$ & 0.00 \\
\hline \multicolumn{7}{|l|}{ Regimen } \\
\hline Olanzapine vs Aprepitant & 0.92 & $0.29-2.92$ & 0.89 & 0.81 & $0.43-1.52$ & 0.51 \\
\hline \multicolumn{7}{|l|}{ rs1045642 } \\
\hline TT vs Non-TT & 0.73 & $0.08-6.52$ & 0.78 & 4.98 & $1.83-13.56$ & 0.00 \\
\hline \multicolumn{7}{|l|}{ rs2032582 } \\
\hline CC vs Non-CC & 1.33 & $0.39-3.66$ & 0.65 & 1.84 & $0.91-3.70$ & 0.09 \\
\hline
\end{tabular}

generally consistent with a Phase III clinical study abroad of a triple antiemetic regimen of $10 \mathrm{mg}$ olanzapine or aprepitant for CINV prevention. ${ }^{13}$ Our study confirmed that $5 \mathrm{mg}$ olanzapine was comparable to aprepitant in efficacy.

A randomized, double-blind controlled study conducted by Suthinee et al using aprepitant with two doses of 5 and $10 \mathrm{mg}$ of olanzapine in a triple antiemetic regimen supported the present study's results. ${ }^{14}$ However, a study by Rumyantsev et al in 2019 showed that $5 \mathrm{mg}$ of olanzapine was more effective than aprepitant for $\mathrm{CR}$ and control of nausea $(P<0.05)$. One of the reasons for the discrepancy might be the small number of patients and using extra day of olanzapine in the former study, which suggested that extending the application duration of olanzapine might improve the efficacy for CINV. The other reason might be that the overlap of acute- and delayedphase of CINV due to the multi-day administration pattern of cisplatin in our study and the change in nausea patterns might lead to a higher TP rate making it more difficult to detect the differences. ${ }^{15}$

The CR rate in the present study was higher than that of the previous study. First, the fractionated dosing pattern of cisplatin was adopted in this study. The previous study confirmed a higher CR rate with multi-day chemotherapy than with single-day chemotherapy. ${ }^{16}$ Second, the vast majority of patients in the former study were young women, while the present study had more older and male patients, and being both young and female were high-risk factors for CINV, thus possibly leading to a higher CR rate. $^{17}$
In this study, the TP rate in both groups reached over $90 \%$ in the acute phase but was low in the delayed phase, indicating that prevention and treatment of CINV in the delayed phase might be more difficult and deserves clinical attention. As for side effects, the incidence of drowsiness in the olanzapine group was $88.46 \%$ in the present study, which was significantly higher than in the aprepitant group. This result was higher than the incidence of drowsiness in two similar studies, probably due to the different evaluation criteria and the higher proportion of elderly patients in this study. Previous studies have shown that the drowsiness effect of olanzapine is more pronounced in elderly patients. ${ }^{14,18,19}$ However, the incidence of drowsiness in our study was similar to that in another study using $5 \mathrm{mg}$ olanzapine for CINV prevention. ${ }^{20}$

In the present study, gender was correlated with the decrease of TP in the delayed phase, and there was a significantly higher risk for CINV $(P=0.000)$ in the female patients. This was consistent with previous studies and confirmed that women remain at increased risk for CINV in the cisplatin multi-day chemotherapy regimen. ${ }^{14,17}$

The ABCB1-encoded P-glycoprotein transports various heterologous compounds and pumps them out of tissues, participating in the absorption, distribution, metabolism, and excretion of many drugs. ${ }^{6,8}$ The three high-frequency variants in the ABCB1 gene, including the rs1045642, rs2032582, and rs1128503, were investigated in the present study. Univariate analysis revealed that the rs1045642TT genotype in the olanzapine group and the rs2032582GG genotype in the aprepitant group were not 
significantly correlated with the acute TP but were correlated with a decreased delayed TP. Multivariate analysis showed that rs1045642TT genotype was an independent risk factor for delayed CINV. Since some first-generation 5-HT3RA, aprepitant, and glucocorticoids are P-glycoprotein substrates, we hypothesized that the SNP of ABCB1 might affect the P-glycoprotein activity and their activity variants might affect the plasma concentrations of these drugs and thus the efficacy. ${ }^{8,21}$ A Japanese study found that the rs1045642TT genotype was correlated with elevated levels of duodenal ABCB1 mRNA in subjects, which plausibly explained the decreased TP rate in patients with the TT genotype in our study. ${ }^{22}$

A foreign study found that the acute TP rate was higher in patients with the rs1045642TT genotypes than those with the non-TT in the granisetron group $(P=0.04)$. In contrast, this result was not shown in the ondansetron or toltesetron groups. ${ }^{4}$ Zoto et al also found similar results, ${ }^{5}$ which were inconsistent with our results. The relationship between the gene polymorphisms and drug efficacy has shown different results in several studies due to differences in enrollment and intervention protocols. ${ }^{23}$ For example, a meta-analysis assessing the effect of rs1045642 on its substrate's pharmacokinetics yielded opposite results in the white and Asian populations. The blood concentration of digoxin was lower in Caucasian subjects with CC genotype, but higher in Japanese subjects. ${ }^{24}$ This could explain the different results yielded in various studies.

The ABCB1 gene is highly polymorphic in allelic variant frequencies among different ethnic groups, and some genetic variants can affect the individual differences in pharmacokinetics and pharmacodynamics of drugs. $\mathrm{He}$ et al conducted a study with ondansetron administration to Chinese patients with acute myeloid leukemia and found that the rs $1045642 \mathrm{CC}$ was correlated with grade $3 / 4$ vomiting. ${ }^{6}$ Tropisetron was adopted in this study, and it was reported that it might not be affected by the rs 1045642 compared to ondansetron. ${ }^{25}$ This suggested that ABCB1 may also lead to different results if chemotherapeutic and antiemetic drugs were substrates.

The rs 1045642 is not the only gene affecting the ABCB1 polymorphism. The rs2032582GG genotype was found by $\mathrm{He}$ et al to be correlated with acute nausea in the recessive model and with acute vomiting in the dominant model, which was not the same as the results in our study. ${ }^{6}$ In this study, the rs 1045642 locus was correlated with a decreased delayed TP but not the acute TP. This locus is still controversial because three foreign studies have found an increase of TP rates in patients with the rs2032582TT genotype used granisetron, but no correlation was found in the palonosetron group. ${ }^{5,7,8}$

In 2020, Tsuji et al failed to find this correlation in patients using a triple antiemetic regimen containing granisetron. ${ }^{9}$ Therefore, it could be hypothesized that the SNP or unique interactions within multigene traits might be responsible for the expression and phenotype of P-glycoprotein and that individual SNPs have limited predictive power as pharmacogenetic loci. ${ }^{24}$ For the rs1128503 gene, no correlation with CINV was found in our study, which was consistent with most studies. ${ }^{9,10}$ However, Zoto et al found that the acute-phase TP rate was higher in patients with the TT genotype than in other genotypes. $^{5}$

There might be two reasons for this. The first was that although cisplatin is not a substrate for the P-glycoprotein, chemotherapeutic agents as the $\mathrm{ABCB} 1$ substrates might be included in this study, which led to different results. ${ }^{5,8}$ The second was that a triple antiemetic regimen of olanzapine or aprepitant combined with toltestrone and dexamethasone was adopted in our study. In contrast, the aforementioned study included a triple antiemetic regimen of granisetron or ondansetron combined with dexamethasone in a two-combination antiemetic regimen. Studies have confirmed that olanzapine and aprepitant are both substrates of the ABCB1 transporter protein, which might affect the efficacy of antiemetic drugs by influencing the blood concentrations. ${ }^{9,21}$

The DRD2 receptor, encoded by the DRD2 gene, is a dopamine autoreceptor with the mechanism of action to cause nausea and vomiting by inhibiting the adenylate cyclase and altering the cAMP content in neurons. ${ }^{25}$ Olanzapine acts as an antiemetic mainly through competitive antagonism of the DRD2 receptor. The DRD2 mutations may affect the number, distribution, sensitivity, and affinity of DRD2 receptors in brain tissue and affect the efficacy of olanzapine. ${ }^{22,23,25}$ The DRD2 rs1076560 and rs6277 were investigated in this study. Although no significant correlation between the two loci with TP was found, we observed a higher delayed-phase TP rate in patients with the rs1076560CC genotype than in those with the non-CC in the aprepitant group, which was $65.8 \%$ vs $50.8 \%$, and no correlation was found in the olanzapine group.

However, a Japanese study found an increased incidence of CINV in patients with the rs1076560 CC 
genotype. ${ }^{10}$ In addition to the interference of some clinical factors, the above differences might be related to the efficacy evaluation indicators and time. The Japanese study selected the day with the most severe nausea and vomiting within 10 days of the initiation of chemotherapy for evaluation, and the $\mathrm{CP}$ rate should meet nausea $<$ grade 2 and vomiting $<$ grade 1 , which was different from the current common clinical evaluation indicators and might affect the interpretation of the results. For the rs6277 genotype, we failed to find a significant correlation with CINV, which was consistent with the Japanese study. ${ }^{10}$ It has been suggested that the rs6277TT reduces the DRD2 receptor stability by altering the folding structure of mRNA, which may reduce the occurrence of CINV. ${ }^{26}$ This trend was also shown in our study. In both the olanzapine and aprepitant groups, the delayed TP rate was higher in those with the rs6277 non-CC genotype than those with CC, which was $88.9 \%$ vs $52.9 \%$ and $72.7 \%$ vs $54.7 \%$ in the two groups, respectively. The lack of statistical difference might be correlated with the smaller number of cases.

The universal transcription factor IIE1, encoded by the GTF2E1 gene, is essential for transcription initiation through direct and interactive interactions with DNA repair factors. ${ }^{11}$ No correlation between the rs447978 and TP was found in this study. One study showed that this gene was correlated with a reduced incidence of nausea, but no specific allele was reported. ${ }^{11}$ The COMT enzyme encoded by the COMT gene may regulate neurotransmission by metabolizing catecholamines and dopamine and produce an antiemetic effect when the DRD2 receptor is blocked. Thus, the COMT gene polymorphism may affect the occurrence of CINV. ${ }^{27}$ It was shown that the COMT activity in the rs4680GG homozygote was about 3-4 times higher than in the AA homozygote. This was different from our results and those of Mukoyama et al in that the rs4680 was not correlated with CINV. ${ }^{10,28}$ The functional relevance of these genes is clear, and further functional characterization of these SNPs and verification of the correlation with CINV are still needed.

Some limitations of our study are as follows: (1) the concentrations of antiemetic drugs in plasma or cerebrospinal fluid were not measured, and pharmacokinetic data were not available. Thus, the basis of action of each gene polymorphism and the response mechanism of antiemetic drugs could not be elucidated; (2) the sample size used to analyze the correlation between the gene polymorphisms and TP was small. Especially in the acute phase, the ability to show differences in TP rates might be lacking.

The present study confirmed the comparable efficacy of $5 \mathrm{mg}$ of olanzapine and $\mathrm{NK}-1$ receptor antagonist in preventing the cisplatin multi-day chemotherapy induced CINV and revealed that females and the rs1045642TT genotype were independent risk factors for delayed CINV in the northern Chinese population. This provided a scientific basis for subsequent analysis of the risk factors for CINV in Chinese patients. The correlation between the gene polymorphisms and the efficacy of cisplatin multiday chemotherapy-induced nausea and vomiting is influenced by multiple factors, such as ethnicity, emesiscausing drugs, prophylactic regimens, and evaluation indicators, and the results of clinical studies should be interpreted with caution. Exploring more stable markers to predict the incidence and severity of CINV, together with antiemetics' efficacy, is a direction for future investigation.

\section{Data Sharing Statement}

The authors intend to share individual deidentified participant data. Data obtained in the results section: the therapeutic effect of CINV and the relationship between gene polymorphism and complete protection rate of CINV can be shared. Emails could be sent to the address to obtain the shared data: li_qfedu@163.com. We may balance the potential benefits and risks for each request and then provide the data that could be shared. Data will be available for 2 years after publication.

\section{Ethics Approval and Consent to Participate}

This study was conducted with approval from the Ethics Committee of Ordos Central Hospital, Ordos Clinical College of Inner Mongolia Medical University (2019005). This study was conducted in accordance with the declaration of Helsinki. Written informed consent was obtained from all participants.

\section{Funding}

This study was supported by Ordos Health Commission Key discipline Project and Inner Mongolia Medical University Science and Technology Million Project funding.

\section{Disclosure}

The authors report no conflicts of interest in this work. 


\section{References}

1. Hesketh PJ, Bohlke K, Lyman GH. Antiementics: Americian Society of Clinical Oncology Focused Guideline Update. J Clin Oncol. 2016;34:381-386. doi:10.1200/JCO.2015.64.3635

2. Hesketh PJ, Grunberg SM, Herrstedt J, et al. Combined data from two phase III trials of the NK1 antagonist aprepitant plus a 5HT 3 antagonist and a corticosteroid for prevention of chemotherapy-induced nausea and vomiting: effect of gender on treatment response. Support Care Cancer. 2006;14(4):354-360. doi:10.1007/s00520-005-0914-4

3. NCCN Clinical Practice Guidelines in Oncology: Antiemesis. Version 2.2020. Available at https://www.nccn.org/professionals/phy sician_gls/pdf/antiemesis.pdf. Accessed October 12, 2020.

4. Babaoglu MO, Bayar B, Aynacioglu AS, et al. Association of the $\mathrm{ABCB} 13435 \mathrm{C}>\mathrm{T}$ polymorphism with antiemetic efficacy of 5hydroxytryptamine type 3 antagonists. Clin Pharmacol Ther. 2005;78(6):619-626. doi:10.1016/j.clpt.2005.08.015

5. Zoto T, Kilickap S, Yasar U, et al. Improved anti-emetic efficacy of 5-HT3 receptor antagonists in cancer patients with genetic polymorphisms of ABCB1 (MDR1) drug transporter. Basic Clin Pharmacol Toxicol. 2015;116(4):354-360. doi:10.1111/bcpt.12334

6. He H, Yin J, Xu Y, et al. Association of ABCB1 polymorphisms with the efficacy of ondansetron in chemotherapy-induced nausea and vomiting. Clin Ther. 2014;36(8):1242-1252. doi:10.1016/j. clinthera.2014.06.016

7. Tsuji D, Kim Y, Nakamichi H, et al. Association of ABCB1 polymorphisms with the antiemetic efficacy of granisetron plus dexamethasone in breast cancer patients. Drug Metab Pharmacokinet. 2013;28(4):299-304. doi:10.2133/dmpk.DMPK-12-RG-084

8. Tsuji D, Yokoi M, Suzuki K, et al. Influence of ABCB1 and ABCG2 polymorphisms on the antiemetic efficacy in patients with cancer receiving cisplatin-based chemotherapy: a TRIPLE pharmacogenomics study. Pharmacogenomics J. 2017;17(5):435-440. doi:10.1038/tpj.2016.38

9. Tsuji D, Matsumoto M, Kawasaki Y, et al. Analysis of pharmacogenomic factors for chemotherapy-induced nausea and vomiting in patients with breast cancer receiving doxorubicin and cyclophosphamide chemotherapy. Cancer Chemother Pharmacol. 2021;87 (1):73-83. doi:10.1007/s00280-020-04177-y

10. Mukoyama N, Yoshimi A, Goto A, et al. An analysis of behavioral and genetic risk factors for chemotherapy-induced nausea and vomiting in Japanese subjects. Biol Pharm Bull. 2016;39(11):1852-1858. doi:10.1248/bpb.b16-00440

11. Lamba JK, Fridley BL, Ghosh TM, et al. Genetic variation in platinating agent and taxane pathway genes as predictors of outcome and toxicity in advanced non-small-cell lung cancer. Pharmacogenomics. 2014;15(12):1565-1574. doi:10.2217/pgs.14.107

12. Mccrea JB, Majumdar AK, Goldberg MR, et al. Effects of the neurokinin1 receptor antagonist aprepitant on the pharmacokinetics of dexamethasone and methylprednisolone. Clin Pharmacol Ther. 2003;74(1):17-24. doi:10.1016/S0009-9236(03)00066-3

13. Navari RM, Gray SE, Kerr AC. Olanzapine versus aprepitant for the prevention of chemotherapy-induced nausea and vomiting: a randomized phase III trial. J Support Oncol. 2011;9(5):188-195. doi:10.1016/j.suponc.2011.05.002

14. Ithimakin S, Theeratrakul P, Laocharoenkiat A, et al. Randomized, double-blind, placebo-controlled study of aprepitant versus two dosages of olanzapine with ondansetron plus dexamethasone for prevention of chemotherapy-induced nausea and vomiting in patients receiving high-emetogenic chemotherapy. Support Care Cancer.
2020;28(11):5335-5342. doi:10.1007/s00520-020-05380-6

15. Rumyantsev A, Glazkova E, Nasyrova R, et al. Olanzapine (OLN) versus aprepitant (APR) in patients receiving high-emetogenic chemotherapy: final results of randomized Phase II trial. J Clin Oncol. 2019;37(15_suppl):11504. doi:10.1200/JCO.2019.37.15_suppl.11504

16. Li Q, Wang W, Chen G, et al. Evaluation of a neurokinin-1 antagonist in preventing multiple-day cisplatin-induced nausea and vomiting. Open Med (Wars). 2018;13:29-34. doi:10.1515/med-2018-0005

17. Mosa ASM, Hossain AM, Lavoie BJ, et al. Patient-related risk factors for chemotherapy-induced nausea and vomiting: a systematic review. Front Pharmacol. 2020;11:329. doi:10.3389/ fphar.2020.00329

18. Hashimoto H, Abe M, Tokuyama $\mathrm{O}$, et al. Olanzapine $5 \mathrm{mg}$ plus standard antiemetic therapy for the prevention of chemotherapy-induced nausea and vomiting (J-FORCE): a multicentre, randomised, double-blind, placebo-controlled, Phase 3 trial. Lancet Oncol. 2020;21(2):242-249. doi:10.1016/S14702045(19)30678-3

19. Liu L, Ding C, Wang J, et al. Low dose olanzapine can improve delayed nausea and vomiting induced by high emetogenic chemotherapy in female patients with lung cancer. $J$ Oncol. 2017;23 (03):251-253.

20. Abe M, Hirashima Y, Kasamatsu Y, et al. Efficacy and safety of olanzapine combined with aprepitant, palonosetron, and dexamethasone for preventing nausea and vomiting induced by cisplatin-based chemotherapy in gynecological cancer: KCOG-G1301 phase II trial. Support Care Cancer. 2016;24(2):675-682. doi:10.1007/s00520-0152829-z

21. Saiz-Rodríguez M, Belmonte C, Román M, et al. Effect of ABCB1 C3435T polymorphism on pharmacokinetics of antipsychotics and antidepressants. Basic Clin Pharmacol Toxicol. 2018;123 (4):474-485. doi:10.1111/bcpt.13031

22. Nakamura T, Sakaeda T, Horinouchi M, et al. Effect of the mutation (C3435T) at exon 26 of the MDR1 gene on expression level of MDR1 messenger ribonucleic acid in duodenal enterocytes of healthy Japanese subjects. Clin Pharmacol Ther. 2002;71(4):297-303. doi:10.1067/mcp.2002.122055

23. Perwitasari DA, Gelderblom H, Atthobari J, et al. Anti-emetic drugs in oncology: pharmacology and individualization by pharmacogenetics. Int $J$ Clin Pharm. 2011;33(1):33-43. doi:10.1007/s11096-010-9454-1

24. Chowbay B, Li H, David M, et al. Meta-analysis of the influence of MDR1 C3435T polymorphism on digoxin pharmacokinetics and MDR1 gene expression. Br J Clin Pharmacol. 2005;60(2):159-171. doi:10.1111/j.1365-2125.2005.02392.x

25. Janicki PK, Sugino S. Genetic factors associated with pharmacotherapy and background sensitivity to postoperative and chemotherapy-induced nausea and vomiting. Exp Brain Res. 2014;232(8):2613-2625. doi:10.1007/s00221-014-3968-z

26. Fernandez-Rozadilla C, Cazier JB, Moreno V, et al. Pharmacogenomics in colorectal cancer: a genome-wide association study to predict toxicity after 5-fluorouracil or FOLFOX administration. Pharmacogenomics J. 2013;13(3):209-217. doi: $10.1038 /$ tpj.2012.2

27. Hosák L. Role of the COMT gene Val158Met polymorphism in mental disorders: a review. Eur Psychiatry. 2007;22(5):276-281. doi:10.1016/j.eurpsy.2007.02.002

28. Laugsand EA, Fladvad T, Skorpen F, et al. Clinical and genetic factors associated with nausea and vomiting in cancer patients receiving opioids. Eur J Cancer. 2011;47(11):1682-1691. doi:10.1016/j. ejca.2011.04.014 


\section{Publish your work in this journal}

Pharmacogenomics and Personalized Medicine is an international, peer-reviewed, open access journal characterizing the influence of genotype on pharmacology leading to the development of personalized treatment programs and individualized drug selection for improved safety, efficacy and sustainability. This journal is indexed on the American Chemical Society's Chemical Abstracts Service (CAS). The manuscript management system is completely online and includes a very quick and fair peer-review system, which is all easy to use. Visit http://www.dovepress.com/testimonials.php to read real quotes from published authors. 